\title{
Calculations of Perovskite Polar Surface Structures
}

\author{
E. Heifets ${ }^{a *}$, R. I. Eglitis ${ }^{b}$, E. A. Kotomin ${ }^{c, d}$, W.A. Goddard III ${ }^{a}$, \\ and G. Borstel ${ }^{b}$ \\ ${ }^{a}$ Materials and Process Simulation Center, Beckman Institute (139-74), \\ California Institute of Technology, MS 139-74, Pasadena CA 91125, USA \\ ${ }^{b}$ Universität Osnabrück, Fachbereich Physik, D-49069 Osnabrück, Germany \\ ${ }^{c}$ Institute for Solid State Physics, University of Latvia, 8 Kengaraga, Riga LV-1063, Latvia \\ ${ }^{d}$ Max Planck Insitut für Festkörperforschung, Heisenbergstr., 1, D-70569 Stuttgart, Germany
}

\begin{abstract}
Results of calculations for the (110) polar surfaces of three $\mathrm{ABO}_{3}$ perovskites - STO, BTO and LMO - are discussed. These are based on $a b$ initio HartreeFock method and classical Shell Model. Both methods agree well on both surface energies and on near-surface atomic displacements. A novel model of the "zig-zag" surface termination is suggested and analyzed. Considerable increase of the $\mathrm{Ti}-\mathrm{O}$ chemical bond covalency nearby the surface is predicted for STO.
\end{abstract}

\section{INTRODUCTION}

Thin films of $\mathrm{ABO}_{3}$ perovskite ferroelectrics are important for many technological applications, including catalysis, microelectronics, substrates for growth of high $\mathrm{T}_{c}$ superconductors, where surface structure and its quality are of primary importance $[1,2]$. Several $a b$ initio quantum mechanical [3-9] and classical Shell Model (SM) $[10,11]$ theoretical studies were published recently for the (100) surface of $\mathrm{BaTiO}_{3}$ and $\mathrm{SrTiO}_{3}$ crystals (hereafter $\mathrm{BTO}$ and STO). In order to study dependence of the surface relaxation properties on exchange-correlation functionals and localized/plane wave basis sets used in calculations, we performed recently a detailed comparative study based on a number of different quantum mechanical techniques [12-14]. The main conclusion was drawn there that the Hartree-Fock (HF), Density Functional Theory (DFT), and even SM calculations give quite similar results for the atomic structure relaxation and surface energies.

We performed also SM calculations of the atomic relaxation for the polar (110) surfaces of STO and BTO [11]. To our knowledge, only semi-empirical quantum mechanical calculations [15] exist so far for such perovskite surfaces. In this paper, we present a novel, "zig-zag" model for the polar (110) surface termination, and

CP677, Fundamental Physics of Ferroelectrics 2003, edited by P. K. Davies and D. J. Singh

(c) 2003 American Institute of Physics 0-7354-0146-2/03/\$20.00 
(a) O-terminated (011) surface, configuration $\mathbf{A}$ (top view on left and side view on right)
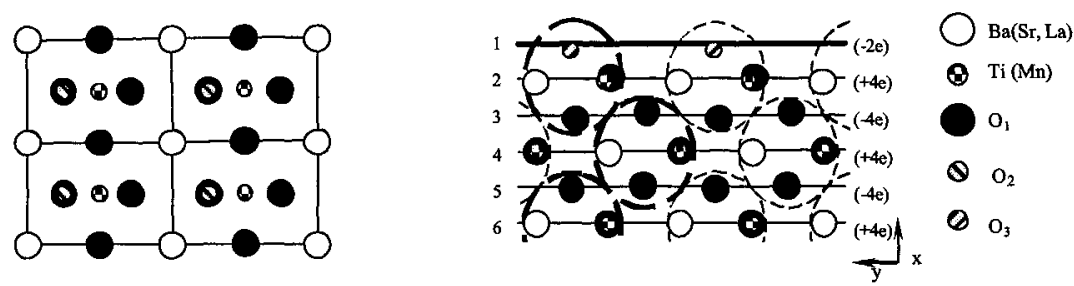

(b) O-terminated (011) surface, configuration B (top view on left and side view on right)
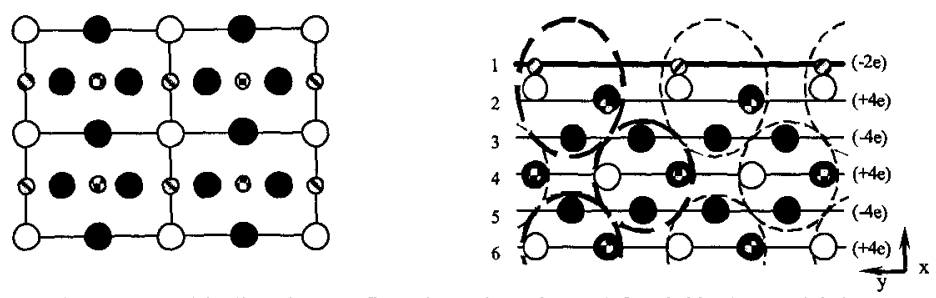

(c) O-terminated (011) surface, configuration C (top view on left and side view on right)
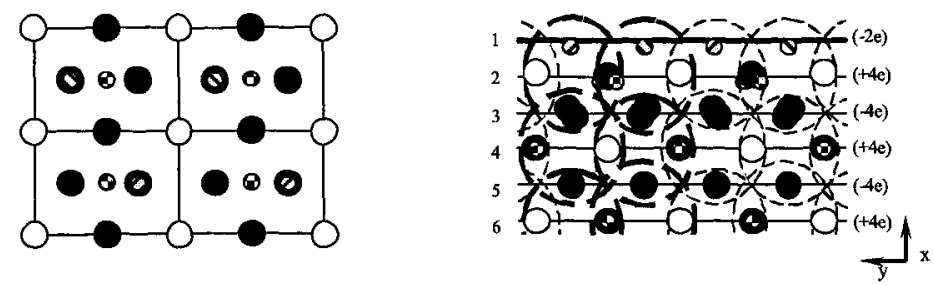

(d) TiO (MnO) -terminated (011) surface

(e) $\mathrm{Sr}(\mathrm{Ba}, \mathrm{La})$ - terminated (011) surface
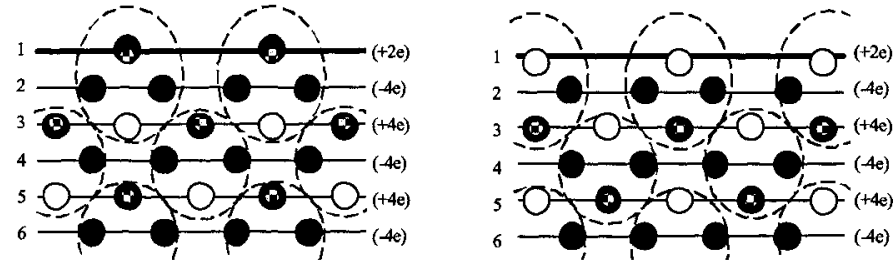

FIGURE 1. The top and side view of the (110) perovskite surfaces. (a), (b), (c) are three possible configurations of $\mathrm{O}$-terminated surface, (d) and (e) same for $\mathrm{TiO}$ - and $\mathrm{Sr}(\mathrm{Ba})$ terminations, see details in the text. 
perform calculations of the relaxed atomic structure of the STO, BTO and $\mathrm{LaMnO}_{3}$ (LMO) (110) surfaces, combining the ab initio HF and classical SM methods.

\section{METHODS AND SURFACE MODELS}

In this study, we restrict ourselves to simulations of $\mathrm{ABO}_{3}$ perovskites in the cubic crystalline phase, stable at high temperatures. Description of SM and its parameterization is available in Ref. [11]. Use of this model permits us to find the atomic relaxation for several hundreds of atoms, surface energies, along with the surface polarization, characterized by dipole moments perpendicular and parallel to the surface. This information is of great importance for analysis of dielectric properties of thin ferroelectric films. We allow atoms in a given number of near-surface planes (varied from 2 to 16) to relax to the minimum of total energy, and then analyze, how the major properties are affected by a number of relaxed planes. This is important since in time-consuming ab initio calculations only 2-3 near-surface planes are typically allowed to relax.

In HF calculations for STO, performed to check accuracy of the SM calculations, we use the CRYSTAL-98 computer code (see [16] and references therein for description of all mentioned techniques), in which both (HF/DFT) types of calculations are implemented on equal grounds. Unlike previous plane-wave calculations, this code uses the localized Gaussian-type basis set. In our simulations we applied the basis set recommended for $\mathrm{SrTiO}_{3}$ [16]. Another advantage of the CRYSTAL-98 code is its treatment of purely $2 \mathrm{D}$ slabs, without an artificial periodicity in the direction perpendicular to the surface, commonly employed in all previous surfaceband structure calculations (e. g., [3,9]). In HF calculations, along with the atomic displacements in several planes near the surface, we calculate effective (Mulliken) atomic charges, bond populations between nearest atoms, characterizing the covalency effects, and dipole, quadrupole moments characterizing atomic polarization and deformation. In particular, the dipole moments $p_{z}$ and $p_{y}$ characterize atomic deformation and polarization along the $z$ axis and the $y$ axis perpendicular and parallel to the surface, respectively.

For optimization of atomic coordinates through minimization of the total energy per unit cell, we use our own computer code that implements the Conjugated Gradients optimization technique with numerical computation of derivatives. Using this code, we optimized the atomic positions in three top layers of a STO slab consisting of seven planes.

The problem of the (110) polar surface modeling is that it consists of charged planes. This is why, if the (110) surface were to be modeled exactly as one would expect after crystal cleavage, it would have in infinite dipole moment perpendicular to the surface, which makes such the surface unstable [17]. To avoid this problem, in our calculations we removed half the $\mathrm{O}$ atoms from from the $\mathrm{O}$-terminated surface, the $\mathrm{Sr}(\mathrm{Ba})$ atoms from the $\mathrm{Ti}$-terminated surface, and both the $\mathrm{Ti}$ and $\mathrm{O}$ atoms from the $\mathrm{Sr}(\mathrm{Ba})$ - terminated surface. As a result, we obtain the surface with 
TABLE 1. Atomic relaxation of three top layers (in percent of the lattice constant) for four terminations, calulated by means of the $a b$ initio HF and Shell Model [11].

\begin{tabular}{|c|c|c|c|c|c|}
\hline $\begin{array}{l}\text { Ti-O terminated } \\
\text { Layer }\end{array}$ & Atom & $\begin{array}{c}\mathrm{SM} \\
\delta \mathrm{z} \\
\end{array}$ & $\delta \mathrm{y}$ & $\begin{array}{r}\mathrm{HF} \\
\delta \mathrm{z} \\
\end{array}$ & $\delta \mathrm{y}$ \\
\hline 1 & $\mathrm{Ti}$ & -5.99 & & -6.49 & \\
\hline 1 & $\mathrm{O}$ & 8.48 & & 6.85 & \\
\hline 2 & $\mathrm{O}$ & -1.72 & & -1.47 & \\
\hline 3 & $\mathrm{O}$ & -4.10 & & -3.85 & \\
\hline 3 & $\mathrm{Ti}$ & 2.14 & & 2.20 & \\
\hline 3 & $\mathrm{Sr}$ & -6.96 & & -5.78 & \\
\hline O-terminated & A-type & & & & \\
\hline 1 & $\mathrm{O}$ & -14.2 & -8.54 & -10.41 & -10.53 \\
\hline 2 & $\mathrm{Ti}$ & -2.37 & -8.27 & -1.36 & -7.71 \\
\hline 2 & $\mathrm{Sr}$ & 4.10 & -10.79 & 2.20 & -7.30 \\
\hline 2 & $\mathrm{O}$ & 5.71 & 8.20 & 6.65 & 6.15 \\
\hline 3 & $\mathrm{O}$ & -11.06 & -11.01 & -7.02 & -7.46 \\
\hline$\overline{\mathrm{O}}$-terminated & B-type & & & & \\
\hline 1 & $\mathrm{O}$ & -2.78 & & -3.95 & \\
\hline 2 & $\mathrm{Ti}$ & -5.14 & & -4.26 & \\
\hline 2 & $\mathrm{Sr}$ & 30.32 & & 22.67 & \\
\hline 2 & $\mathrm{O}$ & 9.68 & & 8.23 & \\
\hline 3 & 0 & -2.41 & & -1.68 & \\
\hline$\overline{\mathrm{O} \text {-terminated }}$ & C-type & & & & \\
\hline 1 & $\mathrm{O}$ & -13.76 & -9.08 & & \\
\hline 2 & $\mathrm{Ti}$ & -4.87 & -5.52 & & \\
\hline 2 & $\mathrm{Sr}$ & 4.31 & 0.0 & & \\
\hline 2 & $\mathrm{O}$ & 1.21 & 0.0 & & \\
\hline 3 & $\mathrm{O}$ & -9.60 & 7.54 & & \\
\hline \multicolumn{6}{|l|}{ Sr-terminated } \\
\hline 1 & $\mathrm{Sr}$ & -19.07 & & -17.38 & \\
\hline 2 & $\mathrm{O}$ & 3.18 & & 2.72 & \\
\hline 3 & $\mathrm{Sr}$ & 4.67 & & 3.95 & \\
\hline 3 & 0 & -0.25 & & -0.21 & \\
\hline 3 & $\mathrm{Ti}$ & -0.89 & & -0.86 & \\
\hline
\end{tabular}


charged planes but a zero dipole moment (before atomic relaxation). The relevant surface cells are built from neutral five-atom elements from three successive planes which are shown as encircled dashed ellipses in Fig.1.

The initial atomic configuration for the $\mathrm{O}$-terminated surface, where every second surface $O$ atom is removed and others occupy the same sites as in the bulk structure, we call asymmetric (A), Fig. 1.a. Since such a removal of half of $O$ atoms disturb the balance of interatomic forces along the surface, we also studied another, symmetric initial surface configuration (B) in which the $\mathrm{O}_{2}$ atom is placed in the middle of the distance between two equivalent $\mathrm{O}$ atoms in the bulk (Fig. 1.b). The A-type surface reveals considerable atomic displacements not only perpendicular to the surface, but also parallel to the surface. Preliminary results for the A-, B-cases were discussed in Ref. [11]. In this paper, we study one more configuration (C) which corresponds to the $2 \times 1$ surface reconstruction where $\mathrm{O}$ atoms are removed in pairs of nearest surface cells in a "zig-zag" way $\left(\mathrm{O}_{2}\right.$ and $\mathrm{O}_{3}$ in Fig. 1.c). In this case, there is no artificial dipole moment parallel to the surface, in contrast to the case A.

The effective charges for Ti and $\mathrm{O}$ ions, both in the bulk and on the (100) surface, calculated by means of the HF and DFT methods [13], are much smaller than formal ionic charges ( 4 e,-2 e, respectively). This arises due to partly covalent nature of the Ti-O chemical bond. In contrast, $\mathrm{Sr}$ charge remains close to the formal charge, +2 e. The Ti-O chemical bond covalency is confirmed by calculated bond populations, which vary from 0.05 e (DFT-LDA) to 0.11 e (HF), dependent on the particular method. Obviously, there is no chemical bonding between any other types of atoms, e.g. $\mathrm{Sr}-\mathrm{O}$ or O-O.

Our atomic displacements in the (100) outermost $\mathrm{SrTiO}_{3}$ planes, obtained by means of various $a b$ initio methods, were analyzed recently $[13,14]$.

In all calculations of the (100) surface energy, that for the SrO termination is only slightly smaller than for the $\mathrm{TiO}_{2}$ termination. Thus, both (100) surfaces can co-exist, in agreement with the experimental observation [13].

\section{MAIN RESULTS}

\section{A Shell Model}

Atomic relaxation of the first three top layers are given in Table 1. SM calculations predict large, $\approx 14 \% \mathrm{a}_{0}$ rumpling for the TiO-terminated surface (the distance along the $z$ axis between $\mathrm{O}$ and $\mathrm{Ti}$ atoms displaced from the first plane in opposite directions). Atomic displacements in the third plane are still considerable, unlike the (100) case. For the Sr-termination, top $\mathrm{Sr}$ atoms are displaced inwards, by $\approx 19 \%$, whereas the $\mathrm{O}$ atoms in the second plane go outwards, by $\approx 3 \%$.

The top $\mathrm{O}$ atom on the $\mathrm{O}$-terminated symmetric surface $(\mathrm{A})$ is strongly displaced inwards, by $\approx 14 \%$, whereas the $\mathrm{Ti}$ atom in the second plane is also displaced inwards, but only by $\approx 2 \%$. Along with the displacements along the $z$ axis per- 
pendicular to the surface, all atoms here reveal also considerable displacements parallel to the surface. This results in the dipole moments $p_{y}$, to be duscussed below.

In contrast, for the symmetric termination $\mathrm{B}$, atoms are displaced only along the $z$ axis and reveal much smaller displacements (e.g., the top $O$ atoms go inward, by $\approx 3 \%$.) However, Sr atoms in the second plane are strongly $(\approx 30 \%)$ displaced outwards the surface. Similar effect was observed by us for the (100) surface [11]. The (110) surface polarization, characterized by the relaxation-induced dipole moments (per surface unit cells) $p_{z}$ and $p_{y}$ perpendicular and parallel to the surface, respectively, is shown in Fig. 2. For the asymmetric termination (A) the surface polarization $p_{z}$ oscillates around $1.1 \mathrm{e} \AA$, with an increase of a number of of relaxed near- surface planes (varied between 2 and 16). This is accompanied by a considerable dipole moment $p_{y}$ parallel to the surface. In contrast, the $p_{z}$ dipole moment for the B-type termination rapidly saturate at 1.13 e $\AA$ whereas $p_{y}$ strives for zero, with an increase of a number of relaxed layers. Lastly, for the "zig-zag" termination $\mathrm{C}, p_{z}$ oscillates around $1.1 \mathrm{e} \AA$ similarly to the asymmetric case (A) but without any dipole moment parallel to the surface. In other words, surface relaxation of cubic perovskite structure leads to the considerable polarization perpendicular to the surface which results from near-surface relaxation. This could considerably affect the dielectric properties of thin films.

Table 2 shows cosiderable difference for surface energies for STO, BTO and LMO (110) surfaces, obtained for two and 16 relaxed layers. (Our calculations show that the (110) surface energy saturates at about 6-8 relaxed layers only, whereas in $a b$ initio calculations only 1-2 layers are typically relaxed.) Unlike the (100) surface, different (110) surface terminations strongly differ in energies. For all three perovskites, the novel, "zig-zag" termination $(\mathrm{C})$ is lowest in surface energy.

\section{B Hartree-Fock Calculations}

The HF-calculated atomic relaxations for STO, shown in Table 1, confirm results of much simpler SM calculations. The agreement for all four termination is remarkable, indeed. This demonstrates that semi-empirical classical calculations with a proper parameterization could serve as a very useful tool for modeling perovskite thin films. Table 2 demonstrates also a good agreement between the HF calculated surface energies and those obtained by means of the SM (comparing in both cases results for two relaxed planes.)

We calculated also the effective atomic charges, dipoles and quadrupole moments for atoms near the surface. For the TiO termination, the Ti atom charge is reduced by $0.14 \mathrm{e}$, as compared to that in the bulk, due to the additional electron charge transfer from $\mathrm{O}$ atoms. The effective charge for $\mathrm{O}$ atoms turns out to be more positive, by $0.11 \mathrm{e}$. The Sr effective charge on the Sr-terinated surface is also reduced by $0.13 \mathrm{e}$, as for the TiO terminated (110) surface, and metal-terminated (100) surfaces. Changes in atomic charges in deeper layers become small. On 


\section{Polarization of O-terminated $\mathrm{SrTiO}_{3}(110)$ Surface}
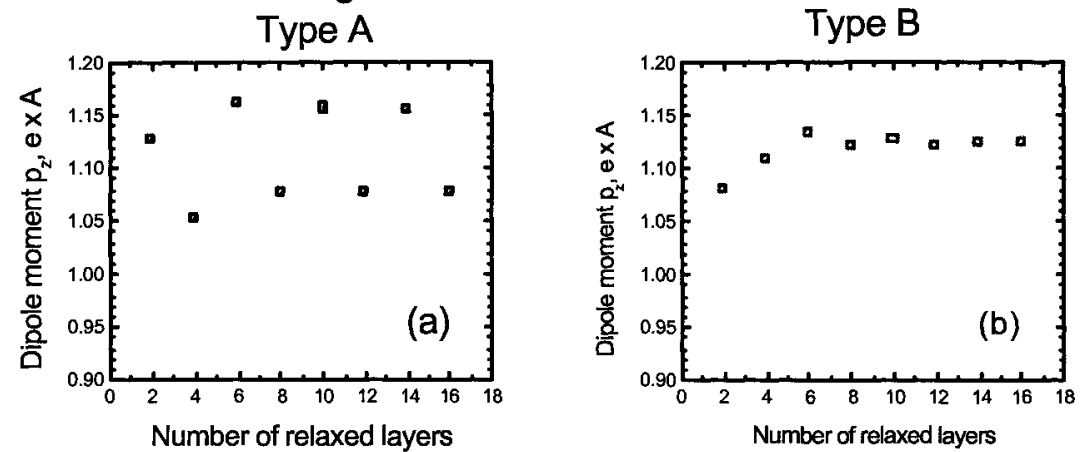

Type A

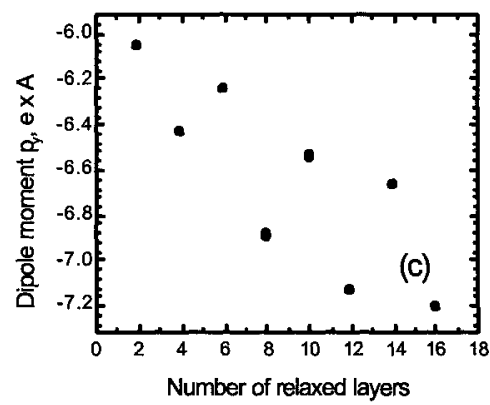

Type B

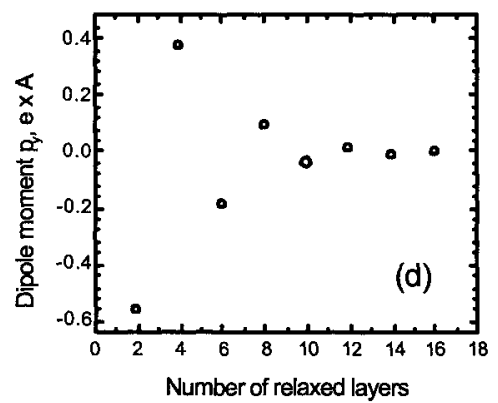

Type C

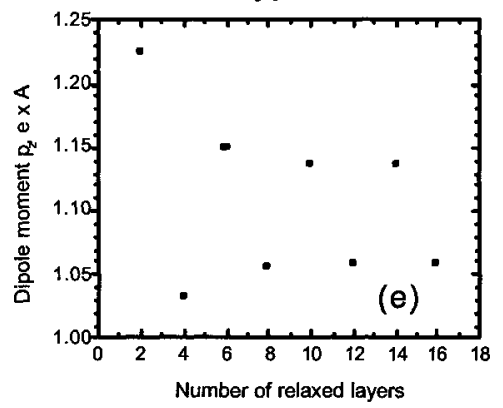

FIGURE 2. Surface polarization and dipole moments perpedicular and parallel to the STO (110) surface with different terminations. 
TABLE 2. Surface energies for four different $O$ (110) terminations shown in Fig.1, as calculated for STO, BTO and LMO perovskites, using the Shell Model (SM) [11] and $a b$ initio HF method (for STO only). SM-2, SM-16 refer to the number of near-surface planes allowed to relax.

\begin{tabular}{llrc} 
Type & HF & SM-2 & SM-16 \\
\hline STO-A-type & 1.40 & 1.54 & 0.92 \\
B & 3.08 & 3.13 & 3.31 \\
C & & 1.63 & 0.76 \\
TiO & 2.10 & 2.21 & 2.36 \\
Sr & 2.97 & 3.04 & 3.37 \\
\hline BTO-A-type & & 1.58 & 1.83 \\
B & & 4.66 & 4.84 \\
C & & 1.84 & 1.82 \\
TiO & & 2.11 & 2.36 \\
Ba & 3.79 & 4.16 \\
LMO-A-type & & 2.59 & 2.06 \\
B & & 4.11 & 4.06 \\
C & & 2.81 & 1.95 \\
\hline
\end{tabular}

the $\mathrm{TiO}$ - and $\mathrm{Sr}$-terminated (110) surfaces both $\mathrm{Ti}$ and $\mathrm{Sr}$ atoms reveal negative dipole moments, directed outwards the surface which means a contraction of the near-surface cations.

The interatomic bond populations for three terminations are given in Table 3. The major effect observed here is a strong increase of the $\mathrm{Ti}-\mathrm{O}$ chemical bonding near the surface as compared to (already large) bonding in the bulk (112 me). The Ti-O bond population for the O-terminated A-type surface is as large as $294 \mathrm{me}$, i.e. by a factor larger than 2 larger than in the bulk. (This factor for the (100) surface was 1.5.) The Ti-O bond population reaches practically the bulk value for atoms in a third plane. An increased Ti-O bond population near the (110) surface obviously does not arise from the surface relaxation. As shows Table 3 , for the $\mathrm{TiO}$ termination and unrelaxed surface $\mathrm{P}[\mathrm{Ti}(\mathrm{I})-\mathrm{O}(\mathrm{II})]=182 \mathrm{me}$, which increases up to 240 me after surface relaxation. Second, for the same interatomic distance, the $\mathrm{Ti}-\mathrm{O}$ bond populations are larger in the direction perpendicular to the surface (182 me) than on-plane (126 me).

\section{CONCLUSIONS}

Our ab initio calculations indicate a considerable increase of the $\mathrm{Ti}-\mathrm{O}$ bond covalency near the (110) surface, much larger than that for the (100) surface. This should have impact on the electronic structure of surface defects (e.g., $F$ centers), as well as affect an adsorption and surface diffusion of atoms and small molecules 
TABLE 3. The A-B bond populations, $P$ (in milli $\mathrm{e}=\mathrm{me}$ ) and the relevant interatomic distances $\mathrm{R}$ (in $\AA$ ) for three different O (110) terminations in STO. I to IV are number of planes enumerated from the surface. The nearest neighbour $\mathrm{Ti}-\mathrm{O}$ distance in the unrelaxed lattice is $1.945 \AA$. Numbers in brackets are bond populations for unrelaxed lattice.

\begin{tabular}{|c|c|c|c|}
\hline Atom A & Atom B & $\mathrm{P}$ & $\mathbf{R}$ \\
\hline \multicolumn{4}{|l|}{ Ti-O terminated } \\
\hline \multirow[t]{2}{*}{$\operatorname{Ti}(\mathrm{I})$} & $\mathrm{O}(\mathrm{I})$ & $176(126)$ & 2.01 \\
\hline & $\mathrm{O}(\mathrm{II})$ & $240(182)$ & 1.81 \\
\hline \multirow[t]{3}{*}{$\mathrm{O}(\mathrm{II})$} & $\mathrm{Ti}(\mathrm{III})$ & $140(130)$ & 1.85 \\
\hline & Sr(III) & -10 & 2.84 \\
\hline & O(III) & -22 & 2.80 \\
\hline \multirow[t]{2}{*}{$\operatorname{Ti}(\mathrm{III})$} & $\mathrm{Sr}(\mathrm{III})$ & 0 & 3.38 \\
\hline & $\mathrm{O}(\mathrm{III})$ & $126(136)$ & 1.96 \\
\hline \multirow[t]{2}{*}{$\operatorname{Sr}(\mathrm{III})$} & $\mathrm{O}(\mathrm{III})$ & -22 & 2.75 \\
\hline & $\mathrm{O}(\mathrm{IV})$ & -24 & 2.64 \\
\hline $\mathrm{Ti}(\mathrm{III})$ & $\mathrm{O}(\mathrm{IV})$ & $108(112)$ & 2.00 \\
\hline $\mathrm{O}(\mathrm{III})$ & $\mathrm{O}(\mathrm{IV})$ & -24 & 2.68 \\
\hline O-terminared & A-type & & \\
\hline \multirow[t]{3}{*}{$\mathrm{O}(\mathrm{I})$} & $\operatorname{Sr}(\mathrm{I})$ & -28 & 2.47 \\
\hline & $\mathrm{Ti}(\mathrm{II})$ & 294 & 1.80 \\
\hline & $\mathrm{O}(\mathrm{II})$ & -26 & 2.90 \\
\hline \multirow[t]{2}{*}{$\mathrm{Sr}(\mathrm{II})$} & $O(I I)$ & -30 & 2.23 \\
\hline & $\mathrm{Ti}(\mathrm{II})$ & 0 & 3.36 \\
\hline \multirow[t]{2}{*}{$\operatorname{Ti}(\mathbf{I I})$} & $\mathrm{O}(\mathrm{II})$ & 90 & 2.04 \\
\hline & $\mathrm{O}(\mathrm{III})$ & 104 & 2.10 \\
\hline $\mathrm{O}(\mathrm{II})$ & O(III) & -28 & 2.85 \\
\hline $\operatorname{Sr}(\mathrm{II})$ & O(III) & -6 & 2.94 \\
\hline \multirow[t]{3}{*}{ O(III) } & $\mathrm{O}(\mathrm{IV})$ & -20 & 2.48 \\
\hline & $\operatorname{Ti}(\mathrm{IV})$ & 110 & 2.00 \\
\hline & $\mathrm{Sr}(\mathrm{IV})$ & -14 & 2.48 \\
\hline O-terminared & B-type & & \\
\hline \multirow[t]{3}{*}{$\mathrm{O}(\mathrm{I})$} & $\operatorname{Sr}(\mathrm{I})$ & -30 & 1.97 \\
\hline & $\mathrm{Ti}(\mathrm{II})$ & 16 & 3.08 \\
\hline & $\mathrm{O}(\mathrm{II})$ & -4 & 3.49 \\
\hline \multirow[t]{2}{*}{$\operatorname{Sr}(\mathrm{II})$} & $\mathrm{O}(\mathrm{II})$ & -20 & 2.81 \\
\hline & $\operatorname{Ti}($ II $)$ & 0 & 3.53 \\
\hline \multirow[t]{2}{*}{$\operatorname{Ti}(\mathrm{II})$} & $\mathrm{O}(\mathbf{I I})$ & 130 & 2.00 \\
\hline & $\mathrm{O}(\mathrm{III})$ & 204 & 1.87 \\
\hline $\mathrm{O}(\mathrm{II})$ & $\mathrm{O}(\mathrm{III})$ & -18 & 2.96 \\
\hline $\operatorname{Sr}(\mathrm{II})$ & $\mathrm{O}(\mathrm{III})$ & 4 & 3.33 \\
\hline \multirow[t]{3}{*}{ O(III) } & $\mathrm{O}(\mathrm{IV})$ & -22 & 2.72 \\
\hline & Ti(IV) & 114 & 1.90 \\
\hline & $\operatorname{Sr}(\mathrm{IV})$ & -22 & 2.72 \\
\hline
\end{tabular}


relevant for catalysis. Atomic displacements calculated by means of classical SM are in surprisingly good agreement with the ab initio HF calculations for STO. The lowest surface energies in all three perovskites studied are found for the novel "zig-zag" $2 \times 1$ reconstructed surface termination. This surface termination reveals no dipole moment parallel to the surface, but considerable dipole moment perpendicular to the surface, which certainly can affect the dielectric properties of thin perovskite films.

\section{ACKNOWLEDGEMENTS}

This study was partly supported by DFG (G. Borstel and R. Eglitis) and European Center of Excellence in Advanced Material Research and Technology in Riga, Latvia (contract No. ICA-I-CT-2000-7007 to EK).

${ }^{*}$ Corresponding author, e-mail: heifets@wag.caltech.edu

\section{REFERENCES}

1. J. F. Scott, Ferroelectric Memories (Springer, Berlin, 2000).

2. M. E. Lines and A. M. Glass, Principles and Applications of Ferroelectrics and Related Materials, Clarendon, Oxford, 1977.

3. J. Padilla and D. Vanderbilt, Surf. Sci. 418, 64 (1998).

4. J. Padilla and D. Vanderbilt, Phys. Rev. B 56, 1625 (1997).

5. B. Meyer, J. Padilla and D. Vanderbilt, Faraday Discussions 114, 395 (1999).

6. F. Cora, and C. R. A. Catlow, Farady Discussions 114, 421 (1999).

7. R. E. Cohen, Ferroelectrics 194, 323 (1997).

8. L. Fu, E. Yashenko, L. Resca, and R. Resta, Phys. Rev. B 60, 2697 (1999).

9. C. Cheng, K. Kunc, and M. H. Lee, Phys. Rev. B 62, 10409 (2000).

10. S. Tinte, and M. G. Stachiotti, AIP Conf. Proc. (ed. R. E. Cohen) 535, 273 (2000).

11. E. Heifets, E. A. Kotomin, and J. Maier, Surf. Sci. 462, 19 (2000).

12. E. A. Kotomin, R. I. Eglitis, J. Maier, and E. Heifets, Thin Solid Films 400, 76 (2001).

13. E. Heifets, R. I. Eglitis, E. A. Kotomin, J. Maier, and G. Borstel, Phys. Rev. B 64, 235417 (2001).

14. E. Heifets, R. I. Eglitis, E. A. Kotomin, J. Maier, and G. Borstel, Surf. Sci. 513, 211 (2002).

15. A. Pojani, F. Finocchi, and C. Noguerra, Surf. Sci. 442, 179 (1999).

16. V. R. Saunders, R. Dovesi, C. Roetti, M. Causa, N. M. Harrison, R. Orlando, and C. M. Zicovich-Wilson, Crystal-98 User Manual (University of Torino, Italy, 1999).

17. P. W. Tasker, J. Phys. C: Solid State Phys. 12, 4977 (1979). 